\title{
The importance of different frailty domains in a population based sample in England
}

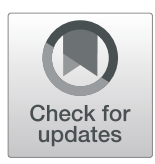

Solveig A. Arnadottir ${ }^{*}$ (D) Julie Bruce ${ }^{2}$, Ranjit Lall², Emma J. Withers², Martin Underwood ${ }^{2}$, Fiona Shaw ${ }^{3}$, Ray Sheridan ${ }^{4}$, Anower Hossain ${ }^{5}$, Sarah E. Lamb ${ }^{2,6}$, on behalf of the Pre-FIT Study Group

\begin{abstract}
Background: The aim was to estimate the prevalence of frailty and relative contribution of physical/balance, nutritive, cognitive and sensory frailty to important adverse health states (falls, physical activity levels, outdoor mobility, problems in self-care or usual activities, and lack of energy or accomplishment) in an English cohort by age and sex.
\end{abstract}

Methods: Analysis of baseline data from a cohort of 9803 community-dwelling participants in a clinical trial. The sample was drawn from a random selection of all people aged 70 or more registered with 63 general practices across England. Data were collected by postal questionnaire. Frailty was measured with the Strawbridge questionnaire. We used cross sectional, multivariate logistic regression to estimate the association between frailty domains and known correlates and adjusted for age. Some models were stratified by sex.

Results: Mean age of participants was 78 years (sd 5.7), range 70 to 101 and $47.5 \%$ (4653/9803) were men. The prevalence of overall frailty was $20.7 \%$ (2005/9671) and there was no difference in prevalence by sex (Odds Ratio 0.98; $95 \%$ Confidence Interval 0.89 to 1.08). Sensory frailty was the most common and this was reported by more men (1823/4586) than women (1469/5056; Odds Ratio for sensory frailty 0.62, 95\% Confidence Interval 0.57 to 0.68). Men were less likely than women to have physical or nutritive frailty. Physical frailty had the strongest independent associations with adverse health states. However, sensory frailty was independently associated with falls, less frequent walking, problems in self-care and usual activities, lack of energy and accomplishment.

Conclusions: Physical frailty was more strongly associated with adverse health states, but sensory frailty was much more common. The health gain from intervention for sensory frailty in England is likely to be substantial, particularly for older men. Sensory frailty should be explored further as an important target of intervention to improve health outcomes for older people both at clinical and population level.

Trial registration: ISRCTN71002650.

Keywords: Frailty, Aging, Population characteristics, Sensation, Hearing, Vision

\section{Background}

Frailty is a theoretical state of vulnerability to adverse health outcomes including death, hospitalization and dependence not accounted for by known disease [1]. The core concept is a loss of homeostatic control at a cellular and organ level, and often at a sub- or pre-clinical level [1]. Frailty is described as a multi-dimensional construct, although there is little consensus on the underlying

\footnotetext{
* Correspondence: saa@hi.is

${ }^{1}$ Department of Physical Therapy, Faculty of Medicine, University of Iceland,

Stapi v/Hringbraut, 101, Reykjavik, Iceland

Full list of author information is available at the end of the article
}

domains [2-4]. Two main frailty models have emerged in last three decades [1, 4], the Fried Phenotypic model of frailty [5], and the Rockwood Cumulative Deficit Model (CDM) [6]. Agreement on a unified model of frailty has been elusive, as have effective population based strategies to minimise frailty.

The Fried model focuses on the role of muscle as the primary reserve organ involved with frailty and hypothesises a direct link between a reduction in muscle mass, strength, metabolic efficiency, related fatigue and exhaustion and slow movement speeds [5]. The CDM is a tally of multiple deficits associated with frailty, including

(C) The Author(s). 2020 Open Access This article is distributed under the terms of the Creative Commons Attribution 4.0 International License (http://creativecommons.org/licenses/by/4.0/), which permits unrestricted use, distribution, and 
mobility, activities of daily living, sensory and cognitive abilities and presence of some chronic conditions [6].

Regardless of specific definition, different frailty models are unified in seeking to understand pathways that minimize old age disability, prolong active life and delay death [2]. Identification of frail community dwelling older adults may reveal early stages and clinically silent vulnerability to environmental challenges. As frailty can be reversible, it is important for society to identify methods to monitor its prevalence amongst aging adults. Considering frailty based on different domains of body function, may be important in identifying groups who can benefit from diverse types of intervention to enhance and maintain functioning and active participation in society.

As part of a large clinical trial investigating the potential of different population screen and treat strategies for geriatric syndromes (falls, fracture and frailty), we assembled a population based cohort in England in 2011 [7]. We used random sampling of 63 general practices to enrol people aged 70 years and older, requesting that they provide data on their health and functional status by post for a minimum of 18 months and allowed us access to their medical records. We selected the Strawbridge questionnaire to measure frailty [8], as at the time it was the only measure validated for postal data collection. The Strawbridge questionnaire collects data on multiple deficits and groups responses into four domains; this measure is consistent with the CDM concept of frailty.

The aim of this analysis was to estimate the prevalence of frailty, and to explore which domains of frailty were most strongly associated with adverse health outcomes for older adults. The underlying premise was to identify the potential for population based interventions in different domains of frailty.

\section{Methods}

\section{Study design and participants}

Sixty-three general practices from South-West (Devon), Central (Warwickshire/Herefordshire, Cambridge, Worcestershire, Birmingham and Black Country) and Northern England (Newcastle) identified a random sample of community dwelling people aged at least 70 years from their practice lists. People with known terminal illness and life expectancy of less than 6 months were excluded by general practitioners. Between September 2011 and June 2014, potential participants were invited to the clinical trial and completed a baseline postal questionnaire. Practices were asked to provide different practice level fall prevention strategies, but participants were not informed of specific interventions. All practices provided brief postal advice on falls prevention and some practices undertook further screening and intervention (the details are reported elsewhere [7] as we report only baseline data here). The study was approved by the National Research Ethics committee (REC 10/H0401/36). Written informed consent was obtained from all participants.

\section{Variables \\ Frailty assessment}

To assess frailty, we used the 1990's Strawbridge questionnaire [8] which was based on an early model of frailty, where the underpinning concept was, vulnerability to environmental challenge based upon complex underlying problems. Building on former studies [9-11], the authors combined impairments across four domains of body function into a single outcome and created a multi-dimensional frailty questionnaire. The Strawbridge questionnaire is a relatively simple and user friendly instrument, and although its reliability has not been reported [4], it has established validity for postal administration and self-completion in older communitydwelling adults $[4,8,12,13]$.

The Strawbridge questionnaire [8] includes 16 items that assess frailty across four domains (physical, nutritional, cognitive, and sensory). Four items represent the physical domain (sudden loss of balance, weakness in arms, weakness in legs, dizzy when stand up quickly), two items represent the nutritive domain (loss of appetite, unexplained weight loss) and four items represent the cognitive domain (difficulty paying attention, trouble finding the right word, difficulty remembering things, forgetting where put things). The final six items represent difficulties in the sensory domain (reading newspapers, recognizing a friend across the street, reading signs at night, hearing over the phone, hearing a normal conversation, hearing a conversation in a noisy room). Our mode of administration, scoring and the final Strawbridge frailty classification was according to the original instructions [8]. Thus, for each of the 16 items, participants self-reported if they had experienced problems over the past 12 months and responses were scored: 1 (rarely or never), 2 (sometimes), 3 (often) and 4 (very often). Participants scoring $\geq 3$ (often or very often) on at least one item in any domain were considered to have impairment/frailty within that domain. Participants were classified as frail (overall frailty) if they reported impairments in two or more domains. Missing values were not replaced, and the scoring rules are such that impairment and frailty can be assessed despite some missing values for individual items.

\section{Sociodemographic characteristics, health and functioning}

We collected self-report data on sex, age, ethnic group, marital status, living arrangements, age leaving full time education (years), height (feet and inches or metres), weight (stones and pounds or kilograms), cognition 
(clock draw test), self-rated health (SRH), chronic diseases and health conditions (angina/heart trouble; anxiety/depression; cancer; arthritis; chronic lung disease; dementia; diabetes; osteoporosis; Parkinson's disease; urinary incontinence; stroke (right/left side)). Body Mass Index $\left(\mathrm{BMI} \mathrm{kg} / \mathrm{m}^{2}\right)$ was calculated using weight and height converted to metric units if appropriate. We used a clock-drawing test [14] to assess global cognition (zero to six point scale whereby higher scores equate to better cognitive ability). SRH was scored on a five point scale (excellent, very good, good, fair, poor) from the 12-Item Short-Form Health Survey (SF-12) [15]. Responses for SRH were collapsed into three categories, $1=$ excellent or very good; 2 = good; 3 = fair or poor.

\section{Adverse health states}

We asked seven questions about adverse health states. Falls were defined using an internationally agreed definition, by recall over the previous 12 months [16]. Participants were asked "are you able to get out and about on foot outside the house" and "on average how many hours a day do you spend walking?" We classified people as having poor outdoor mobility if they were unable to get out and about on foot outside the house unaided. Those walking less than $1 \mathrm{~h}$ per day were considered to have low physical activity. We used the three-level version of the EuroQol five-dimensional questionnaire (EQ5D-3L) [17] to identify participants with limitation in self-care (i.e. some problem or inability to wash or dress themselves), and restricted participation was defined as having some problems or being unable to perform usual activities.

Finally, we used the following two questions from the Short Form 12 Health Survey (SF-12) instrument [15]. We asked, "how much of the time during the past four weeks did you have a lot of energy?" and classed those with no or little energy as having low energy. We asked, "during the past four weeks, have you accomplished less than you would like as a result of your physical health?" and used responses to identify participants who accomplished less than they liked, all or most of the time.

\section{Statistical analysis}

We present descriptive statistics using means, standard deviations (sd) and/or frequency distributions and proportions. Prevalence of frailty by each domain and overall frailty were compared by sex using odds ratio (OR) and $95 \%$ confidence intervals $(95 \% \mathrm{CI})$, with and without adjustment for age. We analysed frailty prevalence by sex (Pearson's chi-square test) in the following age groups: $70-74,75-79,80-84,85-89,90+$ years. We used multivariate logistic regression to estimate the independent contribution of different domains of frailty to adverse health states with adjustment for age. We selected factors for inclusion in multivariate models using univariate analysis and a $P$ value of $<0.1$. Statistical significance in the final models was set at $P<0.05$. Stata/SE (version 15.1) was used for all statistical analyses.

\section{Results}

\section{Participant characteristics}

A total of 29,010 people was invited to take part in the clinical trial, of these 9803 provided valid data and consent $($ response rate $=33.8 \%$; 9803/29010).

Table 1 presents descriptive characteristics of responding participants. Age ranged from 70 to 101 years and 47.5\% (4653/9803) were men. On average, men were slightly younger, more likely to be married or cohabiting, less likely to be living alone and were less likely to report adverse health outcomes than women.

\section{Prevalence of frailty}

Based on observed values, frailty status was identified for 9671/9803 (98.7\%) of the participants. Due to some missing values across individual items, frailty status was missing for $1.3 \%$ of participants. Completion rate by each frailty domain varied, hence the denominator varies. Figure 1 demonstrates frailty across successive age bands by sex. Sensory frailty was more prevalent in men than women across all age bands, although this margin narrowed in those over 90 years of age.

The prevalence of overall frailty was 20.7\% (2005/ 9671), and there was no difference in overall frailty between men and women (960/4592, 20.9\% versus 1045/ 5079, 20.6\%; respectively). The unadjusted odds ratio (OR) for overall frailty, with men as reference, was 0.98 (95\% CI 0.89-1.08) and this estimate did not change when age-adjusted. Sensory frailty was more prevalent in men $(1823 / 4587,39.7 \%)$ than women $(1469 / 5056$, $29.1 \%$ ) whilst physical frailty was more prevalent in women $(1223 / 5098,24 \%)$ compared to men $(881 / 4607$, $19.1 \%)$. Nutritive frailty was almost double in women (266/5101, 5.2\%) compared to men (129/4599, 2.8\%). Cognitive frailty was similar between men $(808 / 4612$, $17.5 \%)$ and women $(876 / 5110,17.1 \%)$. Table 2 presents more detailed prevalences and odds of frailty by sex.

\section{Association with adverse health states}

In all participants, the overall frailty was strongly associated with having fallen in the last year, poor outdoor mobility, lower physical activity level, problems with self-care, restricted participation in usual activities, having less energy and lower accomplishment. For both sexes, this association was independent of age and varied slightly depending on the adverse health state (Table 3). 
Table 1 Characteristics of participants

\begin{tabular}{|c|c|c|c|c|c|}
\hline Characteristics & $n^{a}$ & $\begin{array}{l}\text { Total } \\
(N=9803)\end{array}$ & $\begin{array}{l}\text { Men } \\
(n=4653)\end{array}$ & $\begin{array}{l}\text { Women } \\
(n=5150)\end{array}$ & $P$ value \\
\hline Age, $M \pm s d(\min -\max )$ & 9803 & $\begin{array}{l}77.9 \pm 5.7 \\
(70.0-101.0)\end{array}$ & $\begin{array}{l}77.7 \pm 5.6 \\
(70.0-100.7)\end{array}$ & $\begin{array}{l}78.1 \pm 5.8 \\
(70.0-101.0)\end{array}$ & $<0.001$ \\
\hline Age category, n (\%): & 9803 & & & & 0.003 \\
\hline $70-74$ & & $3670(37.5)$ & 1795 (38.6) & $1875(36.4)$ & \\
\hline $75-79$ & & $2885(29.4)$ & $1376(29.6)$ & $1509(29.3)$ & \\
\hline $80-84$ & & $1951(19.9)$ & 909 (19.5) & $1042(20.2)$ & \\
\hline $85-89$ & & $983(10.0)$ & $441(9.5)$ & $542(10.5)$ & \\
\hline $90+$ & & $314(3.2)$ & $132(2.8)$ & $182(3.6)$ & \\
\hline Ethnic group, white & 9725 & $9630(99.0)$ & 4565 (98.6) & 5065 (99.4) & $<0.001$ \\
\hline Married or cohabiting, n (\%) & 9765 & $6170(63.2)$ & $3638(78.5)$ & $2532(49.3)$ & $<0.001$ \\
\hline Living alone, n (\%) & 9745 & $3217(33.0)$ & $902(19.5)$ & $2315(45.2)$ & $<0.001$ \\
\hline Age when left full time education, $M \pm s d$ (min-max) & 9648 & $\begin{array}{l}16.8 \pm 4.7 \\
(10.0-79.0)\end{array}$ & $\begin{array}{l}16.9 \pm 4.7 \\
(10.0-79.0)\end{array}$ & $\begin{array}{l}16.7 \pm 4.6 \\
(10.0-78.0)\end{array}$ & 0.020 \\
\hline Body Mass Index (BMI), kg/m², M $\pm s d$ (min-max) & 9480 & $\begin{array}{l}26.5 \pm 4.6 \\
(11.6-57.5)\end{array}$ & $\begin{array}{l}26.6 \pm 4.2 \\
(12.8-49.6)\end{array}$ & $\begin{array}{l}26.3 \pm 5.0 \\
(11.6-57.5)\end{array}$ & 0.006 \\
\hline Clock-drawing, ${ }^{\mathrm{b}} \mathrm{M} \pm \mathrm{sd}$ (min-max) & 9621 & $5.5 \pm 0.9(0-6)$ & $\begin{array}{l}5.6 \pm 0.9 \\
(0-6)\end{array}$ & $\begin{array}{l}5.5 \pm 1.0 \\
(0-6)\end{array}$ & 0.009 \\
\hline Clock-drawing, score of $6, n(\%)$ & 9621 & $6865(71.4)$ & $3279(71.8)$ & $\begin{array}{l}3586 \\
(71.0)\end{array}$ & 0.144 \\
\hline Self-rated health, n (\%) & 9718 & & & & $<0.001$ \\
\hline Excellent or very good & & $4314(44.4)$ & $2175(47.1)$ & $2139(41.9)$ & \\
\hline Good & & $3470(35.7)$ & $1570(34.0)$ & $1900(37.2)$ & \\
\hline Fair or poor & & $1934(19.9)$ & $870(18.9)$ & $1064(20.9)$ & \\
\hline \multicolumn{6}{|l|}{ Health conditions, n (\%): } \\
\hline Angina or heart troubles & 9342 & $2697(28.9)$ & $1493(33.2)$ & $1204(24.9)$ & $<0.001$ \\
\hline Anxiety, depression, other & 9116 & $1114(12.2)$ & $412(9.4)$ & $702(14.8)$ & $<0.001$ \\
\hline Arthritis (RA or OA) & 9405 & $4403(46.8)$ & $1643(37.0)$ & $2760(55.7)$ & $<0.001$ \\
\hline Cancer (active) & 9042 & $1316(14.6)$ & $696(15.9)$ & $620(13.3)$ & $<0.001$ \\
\hline Chronic lung disease & 9030 & $641(7.1)$ & $344(7.9)$ & $297(6.4)$ & 0.005 \\
\hline Dementia & 8885 & $68(0.8)$ & $46(1.1)$ & $22(0.5)$ & 0.001 \\
\hline Diabetes & 9135 & $1403(15.4)$ & $770(17.5)$ & $633(13.4)$ & $<0.001$ \\
\hline Osteoporosis & 9012 & $1172(13.0)$ & $195(4.6)$ & $977(20.7)$ & $<0.001$ \\
\hline Parkinson's disease & 8867 & $93(1.1)$ & $58(1.3)$ & $35(0.8)$ & 0.006 \\
\hline Urinary incontinence & 8989 & $933(10.4)$ & $396(9.2)$ & $537(11.5)$ & $<0.001$ \\
\hline Stroke, with affected side & 452 & $174(38.5)$ & 95 (39.9) & 79 (36.9) & 0.513 \\
\hline \multicolumn{6}{|l|}{ Adverse health states, n (\%): } \\
\hline At least one fall in past year & 9737 & $3150(32.4)$ & $1382(29.9)$ & $1768(34.6)$ & $<0.001$ \\
\hline No walking or less than $1 \mathrm{~h} /$ day spent walking & 9754 & $2566(26.3)$ & $1227(26.5)$ & $1339(26.2)$ & 0.733 \\
\hline Unable to get unaided, out and about on foot outside the house & 9763 & $1952(20.0)$ & $702(15.1)$ & $1250(24.4)$ & $<0.001$ \\
\hline Some problems or unable to wash or dress oneself & 9710 & $931(9.6)$ & $418(9.1)$ & $513(10.1)$ & 0.094 \\
\hline Some problems or unable to perform usual activities & 9708 & $3108(32.0)$ & $1295(28.1)$ & $1813(35.6)$ & $<0.001$ \\
\hline Does not have a lot of energy, all or most of the time & 9685 & $5038(52.0)$ & $2152(46.7)$ & $2886(56.8)$ & $<0.001$ \\
\hline Accomplishes less than likes, all or most of the time, due to physical health & 9682 & $1375(14.2)$ & $806(15.9)$ & $569(12.4)$ & $<0.001$ \\
\hline
\end{tabular}

${ }^{\mathrm{a}}$ Number of participants ( $\mathrm{n}$ ), differs between rows due to missing values; ${ }^{\mathrm{b}}$ Clock-drawing test [14] results indicate a global cognitive function. Scores can range from 0 to 6 and higher score indicates better cognitive function; ${ }^{C}$ Continuous variables: $P$ value for t-test (Age, Age when left full time education, BMI, Clockdrawing); Ordinal variables: $P$ value for Mann-Whitney $\mathrm{U}$ test (Age category, Self-rated health); Binary variables: $P$ value for chi-square test (all other variables) 


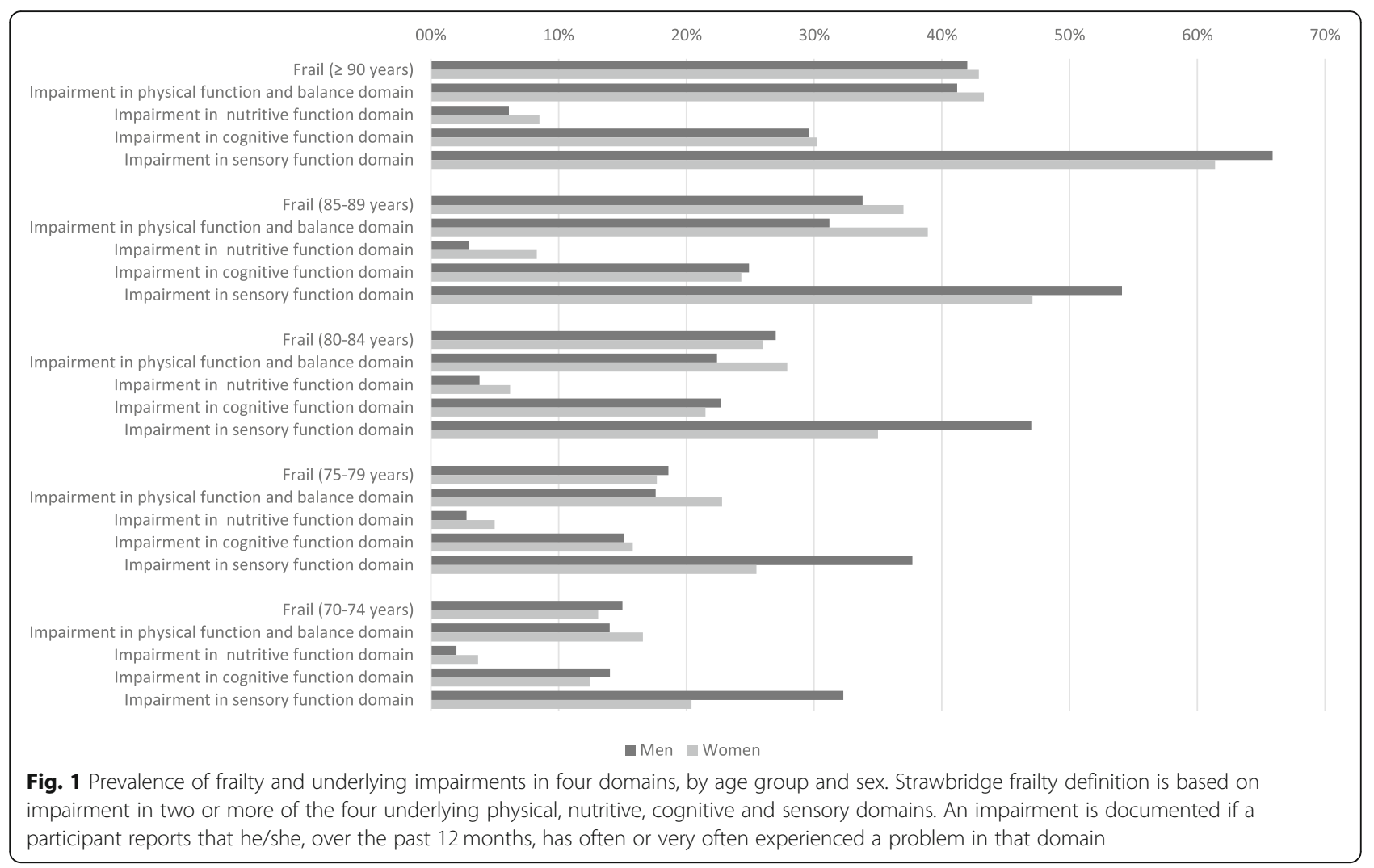

Table 3 also presents the association between each frailty domain and each adverse health state. Overall, physical frailty was strongly associated with all adverse health states. The odds ratio varied across health states, from OR 9.43 (95\% CI 8.34-10.66) for problems with restricted participation in usual activities to OR 2.32 (95\% CI 2.07-2.60) for decreased physical activity level. Nutritive frailty was also significantly associated with all adverse health states and the association varied from OR 5.06 (95\% CI 3.43-7.46) for lower energy to OR 1.84 (95\% CI 1.47-2.29) for falls. Cognitive and sensory frailty demonstrated smaller but consistent associations across the adverse health states. The only health state not associated with cognitive frailty was outdoor mobility. Sensory frailty was associated with all states, although the association with poor outdoor mobility and limitation in self-care was only statistically significant amongst women. Other patterns of association were comparable in both women and men.

\section{Discussion}

In this analysis of baseline data from a cohort of older adults recruited to a falls prevention study, we found that the overall prevalence of frailty was $20.7 \%$ using the Strawbridge questionnaire, with no difference between men and women. Sensory impairments were the most common, particularly common in men, and had modest but important associations with most adverse health states. Physical and nutritive frailty were less prevalent, but more common in women, and were also strongly associated with adverse health states. The findings demonstrate that impairments contributing to frailty are a substantial problem in community dwelling older people in England.

Frailty prevalence amongst older community-dwelling adults has been shown to vary depending on definition, age and health of study cohorts [18]. The underlying definition influences the choice of specific items included in frailty assessments, and hence frailty prevalence. In one large French cohort using the Strawbridge questionnaire in people aged 58 to 73 years [12], the authors demonstrated how inclusion of a sensory domain in a frailty screening instrument increases frailty prevalence, compared to when the sensory domain was excluded. The Strawbridge questionnaire is weighted towards sensory impairment, with the inclusion of six items on vision and hearing (compared to four physical, four cognitive and two nutritive). This allows exploration of the potential contribution of sensory deficits to health and functioning [19]. One sensory question asks about difficulty in understanding speech in challenging environments, such as a noisy room. This 'listening-in-noise' difficulty has been identified in younger people with normal or near-normal hearing thresholds [20] and may be 
Table 2 Prevalence and odds of frailty, impairments in frailty domains by Strawbridge item

\begin{tabular}{|c|c|c|c|c|c|}
\hline \multirow[t]{2}{*}{ Variables based on the Strawbridge questionnaire } & \multicolumn{3}{|c|}{$\begin{array}{l}\text { Prevalence of frailty and impairment } \\
\text { by domain } \\
N(\%)\end{array}$} & \multicolumn{2}{|c|}{$\begin{array}{l}\text { Odds Ratio }{ }^{\text {a }} \text { (95\% Confidence Intervals) } \\
\text { (comparing women to men, men as } \\
\text { reference) }\end{array}$} \\
\hline & $\begin{array}{l}\text { All participants } \\
N=9803\end{array}$ & $\begin{array}{l}\text { Men } \\
n=4653\end{array}$ & $\begin{array}{l}\text { Women } \\
n=5150\end{array}$ & Crude & Adjusted for age \\
\hline Frail $^{\text {b }}$ (data available for $n=9671$ ) & $2005(20.7 \%)$ & $960(20.9 \%)$ & $1045(20.6 \%)$ & $0.98(0.89-1.08)$ & $0.94(0.85-1.04)$ \\
\hline Impairment in physical frailty domain ${ }^{c}(n=9705)$ & $2104(21.7 \%)$ & $881(19.1 \%)$ & $1223(24.0 \%)$ & $1.33(1.21-1.47)^{*}$ & $1.30(1.18-1.44)^{*}$ \\
\hline Sudden loss of balance $(n=9739)$ & $643(6.6 \%)$ & $244(5.3 \%)$ & $399(7.8 \%)$ & $1.52(1.29-1.79)^{*}$ & $1.46(1.24-1.73)^{*}$ \\
\hline Weakness in arms $(n=9721)$ & $799(8.2 \%)$ & $297(6.4 \%)$ & $502(9.8 \%)$ & $1.58(1.36-1.84)^{*}$ & $1.55(1.34-1.80)^{*}$ \\
\hline Weakness in legs $(n=9729)$ & $1457(15.0 \%)$ & $630(13.7 \%)$ & $827(16.2 \%)$ & $1.22(1.09-1.36)^{*}$ & $1.19(1.06-1.33)^{*}$ \\
\hline Dizziness when standing up quickly $(n=9735)$ & $614(6.3 \%)$ & $275(6.0 \%)$ & $339(6.6 \%)$ & $1.122(0.952-1.322)$ & $1.09(0.92-1.29)$ \\
\hline Impairment in nutritive frailty domain $(n=9700)$ & $395(4.1 \%)$ & $129(2.8 \%)$ & $266(5.2 \%)$ & $1.90(1.54-2.36)^{*}$ & $1.86(1.50-2.31)^{*}$ \\
\hline Loss of appetite $(n=9740)$ & $354(3.6 \%)$ & $112(2.4 \%)$ & $242(4.7 \%)$ & $1.99(1.59-2.50)^{*}$ & $1.95(1.55-2.45)^{*}$ \\
\hline Unexplained weight loss $(n=9707)$ & $90(0.9 \%)$ & $31(0.7 \%)$ & $59(1.2 \%)$ & $1.73(1.12-2.67)^{*}$ & $1.68(1.08-2.60)^{*}$ \\
\hline Impairment in cognitive frailty domain $(n=9722)$ & $1684(17.3 \%)$ & $808(17.5 \%)$ & $876(17.1 \%)$ & $0.97(0.88-1.08)$ & $0.95(0.85-1.06)$ \\
\hline Difficulty paying attention ( $n=9732$ ) & $220(2.3 \%)$ & $128(2.8 \%)$ & $92(1.8 \%)$ & $0.64(0.49-0.84)^{*}$ & $0.63(0.48-0.83)^{*}$ \\
\hline Trouble finding the right word $(n=9741)$ & $773(7.9 \%)$ & $333(7.2 \%)$ & $440(8.6 \%)$ & $1.21(1.04-1.40)^{*}$ & $1.18(1.02-1.37)^{*}$ \\
\hline Difficulty remembering things $(n=9747$ ) & $1045(10.7 \%)$ & $539(11.7 \%)$ & $506(9.9 \%)$ & $0.83(0.73-0.94)^{*}$ & $0.80(0.71-0.92)^{*}$ \\
\hline Forgetting where put something $(n=9750)$ & $1104(11.3 \%)$ & $524(11.3 \%)$ & $580(11.3 \%)$ & $1.00(0.88-1.13)$ & $0.97(0.86-1.10)$ \\
\hline Impairment in sensory frailty domain $(n=9643)$ & $3292(34.1 \%)$ & $1823(39.7 \%)$ & $1469(29.1 \%)$ & $0.62(0.57-0.68)^{*}$ & $0.59(0.54-0.64)^{*}$ \\
\hline Difficulty reading a newspaper $(n=9735)$ & $337(3.5 \%)$ & $165(3.6 \%)$ & $172(3.4 \%)$ & $0.94(0.76-1.17)$ & $0.87(0.70-1.09)$ \\
\hline Difficulty recognizing friend across street $(n=9715)$ & $261(2.7 \%)$ & $120(2.6 \%)$ & $141(2.8 \%)$ & $1.06(0.83-1.36)$ & $0.98(0.77-1.26)$ \\
\hline Difficulty reading signs at night $(n=9627)$ & $550(5.7 \%)$ & $216(4.7 \%)$ & $334(6.6 \%)$ & $1.44(1.21-1.72)^{*}$ & $1.39(1.16-1.66)^{*}$ \\
\hline Difficulty hearing over the phone $(n=9727)$ & $1291(13.3 \%)$ & $729(15.8 \%)$ & $562(11.0 \%)$ & $0.66(0.58-0.74)^{*}$ & $0.63(0.56-0.71)^{*}$ \\
\hline Difficulty hearing a normal conversation $(n=9732)$ & $1080(11.1 \%)$ & $615(13.3 \%)$ & $465(9.1 \%)$ & $0.65(0.57-0.74)^{*}$ & $0.63(0.55-0.71)^{*}$ \\
\hline Difficulty hearing conversation in a noisy room $(n=9730)$ & 2919 (30.0\%) & $1683(36.5 \%)$ & $1236(24.2 \%)$ & $0.56(0.51-0.61)^{*}$ & $0.53(0.48-0.58)^{*}$ \\
\hline
\end{tabular}

Number of participants (n), differs between rows due to missing values; ${ }^{\text {a }}$ Odds of frailty, impairment or difficulty, using males as the reference; ${ }^{b}$ Overall frailty is defined as having impairment in $\geq 2$ out of 4 frailty domains; physical, nutritive, cognitive, sensory; ${ }^{c}$ For the four domains (physical, nutritive, cognitive and sensory), impairment is defined as having difficulties in at least one item within the domain; ${ }^{c}$ For each of the 16 items on the Strawbridge questionnaire, participants are asked if they have experienced difficulties over the past 12 months. For the calculation of frailty score, having difficulties on an item is defined as a rating of "often" or "very often" $(\geq 3) ;{ }^{*}$ Indicates a significant difference in frailty scores based on sex

an early predictor of future deficits. This allows for a broader assessment of frailty [12], which again may assist in capturing and assisting people well before they enter potential adverse health states.

In comparison to other studies that have used the Strawbridge questionnaire, our estimate of overall frailty prevalence $(20.7 \%)$ was almost identical to the US Health and Retirement Study (20.3\%) based on a population sample in 2004, aged 65 years and older, without stroke, depression, or moderate to severe cognitive impairments [2]. In the original publication by Strawbridge et al. [8], frailty prevalence in Alameda County US in 1994 was $26.1 \%$, which may reflect inclusion of participants who had moved into an institution. Moreover, the French GAZEL cohort study more recently reported $18.6 \%$ frailty, in a 70-73 years old group, using the Strawbridge questionnaire [12]. Our estimates of impairment rate within each frailty domain are comparable with previous studies $[8,12]$ and the association between frailty and age is well known from other cross-sectional and longitudinal studies $[2,18,21]$.

Our results differ from majority of research which reveal higher prevalence of frailty among women compared to men, but these studies tend to use questionnaires that emphasise the physical and nutritive components of frailty [18, 22-26] or chronic conditions recorded in medical records [27]. The inclusion and number of sensory items makes the Strawbridge questionnaire more sensitive to impairments which are more common in men than women $[8,28]$.

In addition to the prevalence data, our analysis supports the hypothesis that each of the frailty domains are an important construct, as the adverse health states we examined are consistently identified as a high priority by older people [29]. The relationship between physical and nutritive frailty and adverse health states is expected, based on other observations [5]. The relationship between sensory frailty and the adverse health states is 
Table 3 Frailty and impairments by frailty domain, associated with seven adverse health states

\begin{tabular}{|c|c|c|c|}
\hline & All & Men & Women \\
\hline & $\begin{array}{l}\text { Adjusted Odds } \\
\text { Ratio }(95 \% \text { Cl) }\end{array}$ & $\begin{array}{l}\text { Adjusted Odds } \\
\text { Ratio }(95 \% \mathrm{Cl})\end{array}$ & $\begin{array}{l}\text { Adjusted Odds } \\
\text { Ratio (95\% Cl) }\end{array}$ \\
\hline \multicolumn{4}{|l|}{ At least one fall in past year } \\
\hline Frailty $^{a}$ & $2.83(2.55-3.14)$ & $3.10(2.66-3.60)$ & $2.66(2.31-3.07)$ \\
\hline \multicolumn{4}{|l|}{ Frailty domain: ${ }^{b}$} \\
\hline Physical impairment & $2.56(2.30-2.87)$ & $3.05(2.57-3.60)$ & $2.21(1.91-2.56)$ \\
\hline Nutritive impairment & $1.84(1.47-2.29)$ & $1.64(1.11-2.42)$ & $1.93(1.47-2.55)$ \\
\hline Cognitive impairment & $1.62(1.44-1.83)$ & $1.72(1.43-2.05)$ & $1.53(1.30-1.81)$ \\
\hline Sensory impairment & $1.19(1.08-1.31)$ & $1.22(1.05-1.41)$ & $1.22(1.06-1.40)$ \\
\hline \multicolumn{4}{|c|}{ Unable to get unaided, out and about on foot outside the house } \\
\hline Frailty $^{a}$ & $4.18(3.72-4.69)$ & $4.96(4.15-5.94)$ & $3.90(3.34-4.57)$ \\
\hline \multicolumn{4}{|l|}{ Frailty domain: } \\
\hline Physical impairment & $9.11(7.99-10.38)$ & $10.55(8.57-13.00)$ & $8.06(6.80-9.55)$ \\
\hline Nutritive impairment & $2.29(1.80-2.91)$ & $1.85(1.21-2.84)$ & $2.43(1.77-3.33)$ \\
\hline Cognitive impairment & $0.92(0.79-1.07)$ & $0.97(0.76-1.23)$ & $0.88(0.72-1.09)$ \\
\hline Sensory impairment & $1.12(0.99-1.28)$ & $1.18(0.96-1.45)$ & $1.23(1.04-1.47)$ \\
\hline \multicolumn{4}{|c|}{ No walking or less than $1 \mathrm{~h}$ /day spent walking } \\
\hline Frailty $^{a}$ & $2.46(2.21-2.73)$ & $2.54(2.18-2.96)$ & $2.38(2.05-2.76)$ \\
\hline \multicolumn{4}{|l|}{ Frailty domain: ${ }^{b}$} \\
\hline Physical impairment & $2.32(2.07-2.60)$ & $2.42(2.04-2.87)$ & $2.28(1.96-2.65)$ \\
\hline Nutritive impairment & $2.16(1.73-2.70)$ & $2.08(1.42-3.05)$ & $2.25(1.71-2.96)$ \\
\hline Cognitive impairment & $1.24(1.09-1.41)$ & $1.26(1.05-1.52)$ & $1.22(1.02-1.46)$ \\
\hline Sensory impairment & $1.30(1.17-1.45)$ & $1.26(1.08-1.46)$ & $1.31(1.13-1.53)$ \\
\hline \multicolumn{4}{|c|}{ Some problems or unable to wash or dress oneself } \\
\hline Frailty $^{a}$ & $7.26(6.26-8.41)$ & $8.40(6.73-10.48)$ & $6.47(5.30-7.89)$ \\
\hline \multicolumn{4}{|l|}{ Frailty domain:b } \\
\hline Physical impairment & $8.52(7.22-10.05)$ & $9.70(7.57-12.44)$ & $7.61(6.10-9.50)$ \\
\hline Nutritive impairment & $2.25(1.75-2.89)$ & $1.99(1.30-3.06)$ & $2.43(1.78-3.31)$ \\
\hline Cognitive impairment & $1.90(1.61-2.26)$ & $2.35(1.83-3.01)$ & $1.58(1.25-2.00)$ \\
\hline Sensory impairment & $1.31(1.11-1.54)$ & $1.27(0.99-1.64)$ & $1.32(1.05-1.65)$ \\
\hline \multicolumn{4}{|c|}{ Some problems or unable to perform usual activities } \\
\hline Frailty $^{a}$ & $5.90(5.28-6.58)$ & $6.34(5.42-7.43)$ & $5.72(4.91-6.68)$ \\
\hline \multicolumn{4}{|l|}{ Frailty domain: $:^{b}$} \\
\hline Physical impairment & $9.43(8.34-10.66)$ & $9.37(7.79-11.29)$ & $9.21(7.81-10.87$ \\
\hline Nutritive impairment & $2.61(1.99-3.42)$ & $2.56(1.61-4.08)$ & $2.43(1.74-3.40)$ \\
\hline Cognitive impairment & $1.51(1.32-1.73)$ & $1.51(1.24-1.83)$ & $1.50(1.25-1.81)$ \\
\hline Sensory impairment & $1.62(1.46-1.81)$ & $1.71(1.46-2.01)$ & $1.72(1.47-2.01)$ \\
\hline \multicolumn{4}{|c|}{ Does not have a lot of energy, all or most of the time } \\
\hline Frailty $^{a}$ & $5.86(5.15-6.67)$ & $6.14(5.14-7.32)$ & $5.91(4.88-7.17)$ \\
\hline \multicolumn{4}{|l|}{ Frailty domain: ${ }^{b}$} \\
\hline Physical impairment & $6.24(5.41-7.20)$ & $5.82(4.73-7.16)$ & $6.36(5.21-7.77)$ \\
\hline Nutritive impairment & $5.06(3.43-7.46)$ & $6.98(3.41-14.28)$ & $3.98(2.45-6.19)$ \\
\hline Cognitive impairment & $2.27(1.99-2.63)$ & $2.43(2.00-2.95)$ & $2.14(1.75-2.62)$ \\
\hline Sensory impairment & $1.60(1.41-1.72)$ & $1.51(1.31-1.74)$ & $1.91(1.64-2.23)$ \\
\hline
\end{tabular}


Table 3 Frailty and impairments by frailty domain, associated with seven adverse health states (Continued)

\begin{tabular}{|c|c|c|c|}
\hline & All & Men & Women \\
\hline & $\begin{array}{l}\text { Adjusted Odds } \\
\text { Ratio }(95 \% \mathrm{Cl})\end{array}$ & $\begin{array}{l}\text { Adjusted Odds } \\
\text { Ratio }(95 \% \mathrm{Cl})\end{array}$ & $\begin{array}{l}\text { Adjusted Odds } \\
\text { Ratio }(95 \% \mathrm{Cl})\end{array}$ \\
\hline \multicolumn{4}{|c|}{ Accomplishes less than likes, all or most of the time, due to physical health } \\
\hline Frailty $^{a}$ & $6.74(5.94-7.65)$ & $7.64(6.30-9.27)$ & $6.25(5.28-7.39)$ \\
\hline \multicolumn{4}{|l|}{ Frailty domain:b } \\
\hline Physical impairment & $8.27(7.20-9.49)$ & $8.74(7.05-10.83)$ & $7.76(6.47-9.30)$ \\
\hline Nutritive impairment & $3.26(2.54-4.18)$ & $3.64(2.37-5.58)$ & $3.96(2.18-4.02)$ \\
\hline Cognitive impairment & $1.52(1.30-1.78)$ & $1.60(1.26-2.02)$ & $1.46(1.18-1.81)$ \\
\hline Sensory impairment & $1.40(1.22-1.61)$ & $1.40(1.12-1.74)$ & $1.47(1.22-1.78)$ \\
\hline
\end{tabular}

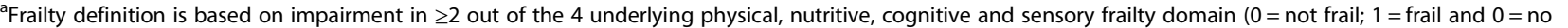
impairment; 1 =impairment); ${ }^{b}$ Each impairment adjusted for the others; All models are based on multivariable logistic regression, adjusting for the effects of age (continuous variable)

consistent with sensory deficits as potential contributors to vulnerability for developing increased dependency [30]. Moreover, Strawbridge's sensory frailty has been shown to predict hospitalisation and, in the same study, both sensory and cognitive frailty were predictive of future disability [12]. Our findings are based on crosssectional data thus a causal pathway between frailty and adverse states cannot be implied from this single time point.

Our results do indicate a potential for future research on population based interventions in different domains of frailty. The Strawbridge questionnaire [8] focuses on limb weakness and balance problems in the physical frailty domain, and exercise interventions can be effective in improving strength and balance [31]. The sensory domain reflects impairments of vision and hearing, and these can be challenging to ameliorate. Yet, provision of services and effective interventions aimed at people with visual and hearing impairments (such as cataract surgery and hearing aids) have the potential to improve social participation and quality of life across large number of older people [32-34]. Therefore, improving treatments for age-associated visual and hearing loss should be a public health priority.

The strength of our work is that the data is drawn from a large population random sample recruited to a clinical trial. A range of GP practices from rural and urban England contributed but a limitation is that these were not a random selection of all English practices. However, they were representative of the anticipated mix of practices in terms of socioeconomic and demographic mix. About one third of older people approached to participate in the study agreed (uptake $34 \%)$. Whilst there were no major differences in age and sex between people agreeing to participate and those not, we have limited data to assess selection bias. The sample was almost identical to the expected age and sex for England [35]. The proportion of people referring to themselves as white was $99 \%$, being higher than estimates from the 2011 census which estimates $98 \%$ at age 90 years and $95 \%$ at age 70 years [35].

The study cohort was assembled for two purposes including future epidemiological research and a cluster randomised controlled trial of screen and treat strategies for geriatric syndromes implemented at the general practice levels. Cohort randomised controlled trials [36] are becoming increasingly common and use a range of different designs which first assemble a cohort and then invite some participants to test interventions using those who are not invited as controls. We used a cluster trial design to assign interventions, with randomisation and intervention at the practice level. Participants were not aware of the interventions being tested by their practice. Baseline data was collected from all participants prior to practice allocation, and hence should not have affected any of the associations or estimates reported in this paper.

While data return and completion were excellent, we may have underestimated the prevalence of sensory and cognitive impairment as these are likely to be associated with questionnaire completion. We asked that participants completed the postal questionnaire themselves, and we accept that it is possible that carers or associates may have completed the questionnaire. This seems unlikely given the pattern of responses we observed and the qualitative narrative that participants provided alongside their questionnaire responses.

Finally, a continuous frailty scale might be more sensitive, but measures which capture the frequency of problems over a longer time period are more predictive than isolated measures of performance [37]. A disadvantage of the Strawbridge assessment of frailty is that it is not widely used, but it remains one of the few instruments that can be collected by postal questionnaire $[4,38]$ and 
it is one of the instruments without items on comorbidity or disability [4]. Although there are numerous frailty scales currently in use, research is needed on their reliability, validity and usefulness in both community and clinical settings $[4,38]$.

\section{Conclusions}

Frailty is a prevalent condition in the population of English people. Sensory frailty is the most common form of frailty and affects more men than women. Although sensory frailty has a more modest association with adverse health states than physical frailty, the potential benefits of clinical and population based intervention to ameliorate sensory frailty should not be overlooked.

\section{Abbreviations}

BMI: Body Mass Index; CDM: Cumulative Deficit Model; Cl: Confidence interval; EQ-5D-3L questionnaire: Three-level version of the EuroQol fivedimensional questionnaire; OR: Odds ratio; SD: Standard deviation; SF-12: 12Item Short-Form Health Survey; SRH: Self Rated Health

\author{
Acknowledgements \\ PreFIT Study Group: \\ Chief Investigator: Professor Sarah E Lamb. \\ Co-investigators (Grant holders): Professor Martin Underwood, Professor \\ Finbarr Martin, Professor Lucy Yardley, Professor Dawn Skelton, Professor \\ Keith Willett, Professor Sandra Eldridge, Dr. Anne-Marie Slowther, Dr. Sarah \\ Duggan. \\ Trial Research Lead: Professor Julie Bruce. \\ Senior Project Manager: Susie Hennings. \\ Trial Co-ordination/Administration: Emma Withers, Susie Hennings, Rhys \\ Mant, Rishpal Rai, Craig Turner, Agata Andrews, Rachael Fearn. \\ Research Fellows/Nurses: Susanne Finnegan, Nicola Walker, Dr. Rachel Potter. \\ Trial statistician: Professor Ranjit Lall. \\ Health Economists: Professor Claire Hulme, Professor Chris Bojke, Dr. Roberta \\ Longo. \\ Clinical Intervention Trainers: Susanne Finnegan, Dr. Katherine Westacott, Dr. \\ Shvaita Ralhan, Dr. Ray Sheridan, Dr. Jonathan Treml. \\ Regional Principal Investigators: Dr. Ray Sheridan (Devon Region), Ms. Jackie \\ Riglin (Cambridge Region); Mr. Harm Gordjin (Warwickshire Region); Dr. \\ Ruma Dutta (Worcestershire Region); Ms. Jo Burns (Hereford Region), Dr. \\ Jonathan Treml (Birmingham \& Black Country Region), Dr. Fiona Shaw, Dr. \\ John Davison (Newcastle Region). \\ Data Programming team: Ade Willis, Chocks Muthiah, Henry Adjei. \\ We extend very grateful thanks to the study participants.
}

\section{Authors' contributions}

SAA analysed and interpreted the data and wrote the manuscript. JB developed the protocol, oversaw the day to day conduct of the study, assisted in data analysis and interpreted the results. AH was a statistician responsible for some analyses. RL was a grant holder and senior statistician. EW was the project manager responsible for co-ordination and data management. MU was a grant holder, senior researcher, provided expertise in primary care. FS and RS contributed to protocol development, provided expertise in geriatric medicine, and commented on the manuscript. SL was chief investigator and study guarantor. All authors read and approved the final manuscript.

\section{Funding}

The PreFIT study is funded by the National Institute of Health Research Technology Assessment Programme (Nir HTA), project number 08/14/41. The views expressed in this publication are those of the authors and not necessarily those of the NIHR or Department of Health. This project benefited from facilities funded by Birmingham Science City Translational Medicine Clinical Research and Infrastructure Trials Platform, with support from Advantage West Midlands (AWM). The trial sponsor is the University of Warwick. The trial started in September 2010 and was funded until 2018.
Professor Sarah Lamb funded by the National Institute for Health Research (NIHR) Collaboration for Leadership in Applied Health Research and Care Oxford at Oxford Health NHS Foundation Trust and NIHR Biomedical Research Centre at the Oxford University Hospitals NHS Trust. Professor Julie Bruce is funded by NIHR Research Capability Funding. The funding bodies had no role in the design, data collection, data analysis, data interpretation, or writing of the manuscript.

\section{Availability of data and materials}

The data that support the findings of this study are available from the Chief Investigator Professor Sarah E Lamb (sarah.lamb@ndorms.ox.ac.uk) upon reasonable requests for data sharing.

\section{Ethics approval and consent to participate}

The study protocol has approval from the National Research Ethics Service (REC reference 10/H0401/36; Protocol V.3.1, 21/May/2013). Written informed consent was obtained from all participants.

\section{Consent for publication}

Not applicable.

\section{Competing interests}

The authors declare that they have no competing interests.

\section{Author details}

${ }^{1}$ Department of Physical Therapy, Faculty of Medicine, University of Iceland, Stapi v/Hringbraut, 101, Reykjavik, Iceland. "Warwick Clinical Trials Unit, Warwick Medical School, University of Warwick, Coventry CV4 7AL, UK. ${ }^{3}$ Older Peoples Medicine, The Newcastle upon Tyne Hospitals NHS Foundation Trust, Freeman Hospital, Freeman Road, High Heaton, Newcastle upon Tyne NE7 7DN, UK. ${ }^{4}$ Geriatric Medicine, Royal Devon and Exeter NHS Foundation Trust, Barrack Road, Exeter, Devon EX2 5DW, UK. ${ }^{5}$ Institute of Statistical Research and Training (ISRT), University of Dhaka, Dhaka, Bangladesh. ${ }^{6}$ Nuffield Department of Orthopaedics Rheumatology \& Musculoskeletal Sciences, University of Oxford, Windmill Road, Oxford OX3 7LD, UK.

Received: 11 October 2018 Accepted: 30 December 2019 Published online: 15 January 2020

\section{References}

1. Clegg A, Young J, lliffe S, Rikkert MO, Rockwood K. Frailty in elderly people. Lancet. 2013;381(9868):752-62.

2. Cigolle CT, Ofstedal MB, Tian Z, Blaum CS. Comparing models of frailty: the health and retirement study. J Am Geriatr Soc. 2009;57(5):830-9.

3. Bergman H, Ferrucci L, Guralnik J, Hogan DB, Hummel S, Karunananthan S, et al. Frailty: an emerging research and clinical paradigm-issues and controversies. J Gerontol A Biol Sci Med Sci. 2007;62(7):731-7.

4. Bouillon K, Kivimaki M, Hamer M, Sabia S, Fransson El, Singh-Manoux A, et al. Measures of frailty in population-based studies: an overview. BMC Geriatr. 2013:13:64.

5. Fried LP, Tangen CM, Walston J, Newman AB, Hirsch C, Gottdiener J, et al. Frailty in older adults: evidence for a phenotype. J Gerontol A Biol Sci Med Sci. 2001;56(3):M146-56.

6. Rockwood K, Song X, MacKnight C, Bergman H, Hogan DB, McDowell I, et al. A global clinical measure of fitness and frailty in elderly people. CMAJ. 2005;173(5):489-95.

7. Bruce J, Lall R, Withers EJ, Finnegan S, Underwood M, Hulme C, et al. A cluster randomised controlled trial of advice, exercise or multifactorial assessment to prevent falls and fractures in community-dwelling older adults: protocol for the prevention of falls injury trial (PreFIT). BMJ Open. 2016:6(1):e009362.

8. Strawbridge WJ, Shema SJ, Balfour JL, Higby HR, Kaplan GA. Antecedents of frailty over three decades in an older cohort. J Gerontol B Psychol Sci Soc Sci. 1998:53(1):S9-16.

9. Buchner DM, Wagner EH. Preventing frail health. Clin Geriatr Med. 1992;8(1):1-17.

10. Fried LP. Conference on the physiologic basis of frailty. April 28, 1992, Baltimore, Maryland, U.S.A. Introduction. Aging (Milano). 1992;4(3):251-2.

11. Guralnik JM, Simonsick EM. Physical disability in older Americans. J Gerontol. 1993;48 Spec No:3-10 
12. Linard M, Herr M, Aegerter P, Czernichow S, Goldberg M, Zins M, et al. Should sensory impairment be considered in frailty assessment? A study in the GAZEL cohort. J Nutr Health Aging. 2016;20(7):714-21.

13. Martinot $P$, Landre B, Zins M, Goldberg M, Ankri J, Herr M. Association between potentially inappropriate medications and frailty in the early old age: a longitudinal Itudy in the GAZEL cohort. J Am Med Dir Assoc. 2018; 19(11):967-973.e3.

14. Shua-Haim J, Koppuzha G, Gross J. A simple scoring system for clock drawing in patients with Alzheimer's disease. J Am Geriatr Soc. 1996;44(3):335.

15. Ware J Jr, Kosinski M, Keller SD. A 12-item short-form health survey: construction of scales and preliminary tests of reliability and validity. Med Care. 1996;34(3):220-33.

16. Lamb SE, Jorstad-Stein EC, Hauer K, Becker C. Prevention of falls network Europe and outcomes consensus group. Development of a common outcome data set for fall injury prevention trials: the prevention of falls network Europe consensus. J Am Geriatr Soc. 2005;53(9):1618-22.

17. Brooks R. EuroQol: the current state of play. Health Policy. 1996;37(1):53-72.

18. Collard RM, Boter H, Schoevers RA, Oude Voshaar RC. Prevalence of frailty in community-dwelling older persons: a systematic review. J Am Geriatr Soc. 2012;60(8):1487-92.

19. Crews JE, Campbell VA. Vision impairment and hearing loss among community-dwelling older Americans: implications for health and functioning. Am J Public Health. 2004;94(5):823-9.

20. Pang J, Beach EF, Gilliver M, Yeend I. Adults who report difficulty hearing speech in noise: an exploration of experiences, impacts and coping strategies. Int J Audiol. 2019;58(12):851-60.

21. Matthews M, Lucas A, Boland R, Hirth V, Odenheimer G, Wieland D, et al. Use of a questionnaire to screen for frailty in the elderly: an exploratory study. Aging Clin Exp Res. 2004;16(1):34-40.

22. Gale CR, Cooper C, Aihie SA. Prevalence of frailty and disability: findings from the English longitudinal study of ageing. Age Ageing. 2015;44(1):162-5.

23. Hubbard RE, Lang IA, Llewellyn DJ, Rockwood K. Frailty, body mass index, and abdominal obesity in older people. J Gerontol A Biol Sci Med Sci. 2010; 65(4):377-81.

24. Puts MT, Lips P, Deeg DJ. Sex differences in the risk of frailty for mortality independent of disability and chronic diseases. J Am Geriatr Soc. 2005;53(1):40-7.

25. Syddall H, Roberts HC, Evandrou M, Cooper C, Bergman H, Aihie SA. Prevalence and correlates of frailty among community-dwelling older men and women: findings from the Hertfordshire cohort study. Age Ageing. 2010;39(2):197-203.

26. Gordon EH, Peel NM, Samanta M, Theou O, Howlett SE, Hubbard RE. Sex differences in frailty: a systematic review and meta-analysis. Exp Gerontol. 2017;89:30-40

27. Clegg A, Bates C, Young J, Ryan R, Nichols L, Ann Teale E, et al. Development and validation of an electronic frailty index using routine primary care electronic health record data. Age Ageing. 2016;45(3):353-60

28. He W, Larsen L, US Census Bureau. American Community Survey Reports, ACS-29, Older Americans with a disability: 2008-2012. Washington, DC: US Government Printing Office; 2014

29. Fried TR, Tinetti ME, lannone L, O'Leary JR, Towle V, Van Ness PH. Health outcome prioritization as a tool for decision making among older persons with multiple chronic conditions. Arch Intern Med. 2011;171(20):1854-6.

30. Grue EV, Ranhoff AH, Noro A, Finne-Soveri $H$, Jensdottir AB, Ljunggren $G$, et al. Vision and hearing impairments and their associations with falling and loss of instrumental activities in daily living in acute hospitalized older persons in five Nordic hospitals. Scand J Caring Sci. 2009;23(4):635-43.

31. Byrne C, Faure C, Keene DJ, Lamb SE. Ageing, muscle power and physical function: a systematic review and implications for pragmatic training interventions. Sports Med. 2016;46(9):1311-32.

32. Lamoureux EL, Pallant JF, Pesudovs K, Rees G, Hassell JB, Keeffe JE. The effectiveness of low-vision rehabilitation on participation in daily living and quality of life. Invest Ophthalmol Vis Sci. 2007;48(4):1476-82.

33. Skelton DA, Howe TE, Ballinger C, Neil F, Palmer S, Gray L. Environmental and behavioural interventions for reducing physical activity limitation in community-dwelling visually impaired older people. Cochrane Database Syst Rev. 2013;6:Cd009233.

34. Ferguson MA, Kitterick PT, Chong LY, Edmondson-Jones M, Barker F, Hoare DJ. Hearing aids for mild to moderate hearing loss in adults. Cochrane Database Syst Rev. 2017;9:Cd012023.

35. Office for National Statistics. CT0702 2011 Census - Age by sex by ethnic group Nat to region. https://www.ons.gov.uk/peoplepopulationandcommunity/ culturalidentity/ethnicity/adhocs/007585ct07022011 censusagesyoabysexbyethnicgroupnattoregion. Accessed 2 Dec 2019.

36. Viksveen P, Relton C, Nicholl J. Benefits and challenges of using the cohort multiple randomised controlled trial design for testing an intervention for depression. Trials. 2017;18(1):308.

37. Lamb SE, McCabe C, Becker C, Fried LP, Guralnik JM. The optimal sequence and selection of screening test items to predict fall risk in older disabled women: the Women's health and aging study. J Gerontol A Biol Sci Med Sci. 2008:63(10):1082-8.

38. Checa-López M, Oviedo-Briones M, Pardo-Gómez A, Gonzales-Turín J, Guevara-Guevara T, Carnicero JA, et al. FRAILTOOLS study protocol: a comprehensive validation of frailty assessment tools to screen and diagnose frailty in different clinical and social settings and to provide instruments for integrated care in older adults. BMC Geriatr. 2019;19(1):86.

\section{Publisher's Note}

Springer Nature remains neutral with regard to jurisdictional claims in published maps and institutional affiliations.
Ready to submit your research? Choose BMC and benefit from:

- fast, convenient online submission

- thorough peer review by experienced researchers in your field

- rapid publication on acceptance

- support for research data, including large and complex data types

- gold Open Access which fosters wider collaboration and increased citations

- maximum visibility for your research: over $100 \mathrm{M}$ website views per year

At BMC, research is always in progress.

Learn more biomedcentral.com/submissions 